\title{
ERROR ANALYSIS OF A SEMIDISCRETE NUMERICAL SCHEME FOR DIFFUSION IN AXIALLY SYMMETRIC SURFACES*
}

\author{
KLAUS DECKELNICK ${ }^{\dagger}$, GERHARD DZIUK ${ }^{\ddagger}$, AND CHARLES M. ELLIOTT $§$
}

\begin{abstract}
We analyze a semidiscrete numerical scheme for approximating the evolution of axially symmetric surfaces by surface diffusion. The fourth order equation is split into two coupled second order problems, which are approximated by linear finite elements. We prove error bounds for the resulting scheme and present numerical test calculations that confirm our analysis.
\end{abstract}

Key words. surface diffusion, finite elements, error estimates, fourth order parabolic equation

AMS subject classifications. $65 \mathrm{~N} 30,35 \mathrm{~K} 55$

DOI. $10.1137 /$ S0036142902405382

1. Introduction. In recent years motion by mean curvature has been extensively studied from the computational point of view. However, the related curvature flow of motion by the surface Laplacian has received far less attention in the numerical analysis literature. The geometrical problem is to find a time-dependent surface $\Gamma(t)$ evolving according to the law of motion

$$
V=\Delta_{\Gamma(t)} \kappa \quad \text { on } \Gamma(t)
$$

where $V$ and $\kappa$ denote, respectively, the normal velocity and the mean curvature of the surface. Our sign convention is that $\kappa$ with respect to the outer normal is positive for spheres. The Laplace-Beltrami or surface Laplacian operator for $\Gamma$ is denoted by $\Delta_{\Gamma}$. This evolution has interesting geometrical properties: if $\Gamma(t)$ is a closed surface bounding a domain $\Omega(t)$, then the volume of $\Omega(t)$ is preserved and the surface area of $\Gamma(t)$ decreases. It is known that for closed curves in the plane or closed surfaces in $\mathbb{R}^{3}$ balls are asymptotically stable subject to small perturbations; see [9], [10]. However, it is also known that topological changes such as pinch-off are possible [11], [13].

Equation (1.1) is referred to as a surface diffusion equation because it models the diffusion of mass within the bounding surface of a solid body. At the atomistic level atoms on the surface move along the surface due to a driving force consisting of a chemical potential difference. For a surface with constant surface energy density the appropriate chemical potential in this setting is the mean curvature $\kappa$. This leads to the flux law

$$
\rho V=-\operatorname{div}_{\Gamma} \mathbf{j}
$$

where $\rho$ is the mass density and $\mathbf{j}$ is the mass flux in the surface, with the constitutive flux law [12], [14]

$$
\mathbf{j}=-D \nabla_{\Gamma} \kappa
$$

*Received by the editors April 9, 2002; accepted for publication (in revised form) April 28, 2003; published electronically November 25, 2003.

http://www.siam.org/journals/sinum/41-6/40538.html

${ }^{\dagger}$ Institut für Analysis und Numerik, Universitatsplatz 2, 39106 Magdeburg, Germany (klaus.deckelnick@mathematik.uni-magdeburg.de).

${ }^{\ddagger}$ Institut für Angewandte Mathematik, Albert Ludwigs Universität Freiburg, Hermann Herder Str. 10, 79104 Freiburg, Germany (gerd@mathematik.uni-freiburg.de).

$\S$ Center for Mathematical Analysis and Applications, School of Mathematical Sciences, University of Sussex, Falmer, Brighton BN1 9QH, UK (c.m.elliott@sussex.ac.uk). 
Here, $D$ is the diffusion constant. From these equations we obtain the law (1.1) after an appropriate nondimensionalization. The notion of surface diffusion is due to Mullins [14] and for a review we refer to [2].

In applications one is interested in the stability of so-called whiskers, which are axially symmetric cylindrical bodies of small diameter with respect to their length; see [15], [3], [1], and [16]. We shall be concerned with an axially symmetric cylindrical body, whose boundary

$$
\Gamma(t)=\left\{\mathbf{x} \in \mathbb{R}^{3} \mid \mathbf{x}=(x, r(x, t) \cos \phi, r(x, t) \sin \phi), x \in[0, L], \phi \in[0,2 \pi]\right\}
$$

evolves by surface diffusion. We assume that the radius $r$ is a smooth positive function, which is periodic in $x$, so that $r(0, t)=r(L, t)$. In these coordinates the mean curvature of $\Gamma(t)$ is

$$
\kappa=\frac{1}{r \sqrt{1+r_{x}^{2}}}-\frac{r_{x x}}{\sqrt{1+r_{x}^{2}}}=\frac{1}{r \sqrt{1+r_{x}^{2}}}-\left(\frac{r_{x}}{\sqrt{1+r_{x}^{2}}}\right)_{x},
$$

while the normal velocity and surface Laplacian of the mean curvature of the surface, respectively, are given by

$$
V=\frac{r_{t}}{\sqrt{1+r_{x}^{2}}}, \quad \Delta_{\Gamma} \kappa=\frac{1}{r \sqrt{1+r_{x}^{2}}}\left(\frac{r \kappa_{x}}{\sqrt{1+r_{x}^{2}}}\right)_{x} .
$$

It follows from these two equations that $r$ satisfies the quasi-linear fourth order parabolic problem

$$
\begin{array}{rlrl}
r_{t} & =\frac{1}{r}\left(\frac{r \kappa_{x}}{\sqrt{1+r_{x}^{2}}}\right)_{x} \quad \text { in } I \times(0, T], \\
r(0, t) & =r(L, t) \quad \text { in }(0, T], \\
\kappa(0, t) & =\kappa(L, t) & \text { in }(0, T], \\
r(\cdot, 0) & =r_{0} & \text { in } I,
\end{array}
$$

where $I=(0, L)$ and $\kappa$ is given by (1.2). The initial function $r_{0}$ is assumed to be periodic and positive.

Our concern in this paper is the analysis of a finite element discretization based on the above natural splitting of the fourth order problem into two coupled second order equations for the radial variable $r$ and the mean curvature $\kappa$. We note that [4] proposed a similar second order splitting scheme and used $R=r^{2}$ and $\kappa$ as the variables. Our principal result is an error estimate for the spatial discretization, which is actually attained in numerical experiments.

The paper is organized as follows: in section 2 we introduce the numerical scheme, prove the local existence and uniqueness of the discrete solution, and formulate our main error estimate. This result is proved in section 3, while section 4 contains numerical tests.

2. The discrete problem. As already mentioned in the introduction, our discretization of (1.3) is based on the idea of splitting the elliptic part, which is of fourth order, into two second order operators. This is similar in spirit to the second order splitting techniques proposed for the numerical approximation of the Cahn-Hilliard 
equation in [8]. To begin, we deduce from (1.2)

$$
r \kappa=\frac{1}{\sqrt{1+r_{x}^{2}}}-r\left(\frac{r_{x}}{\sqrt{1+r_{x}^{2}}}\right)_{x}=\sqrt{1+r_{x}^{2}}-\left(\frac{r r_{x}}{\sqrt{1+r_{x}^{2}}}\right)_{x} .
$$

Thus (1.3) and (2.1) allow the variational formulation

$$
\begin{array}{ll}
\int_{I} r r_{t} \eta d x=-\int_{I} \frac{r \kappa_{x} \eta_{x}}{\sqrt{1+r_{x}^{2}}} d x & \forall \eta \in H_{p e r}^{1}(I), \\
\int_{I} r \kappa \zeta d x=\int_{I} \sqrt{1+r_{x}^{2}} \zeta d x+\int_{I} \frac{r r_{x} \zeta_{x}}{\sqrt{1+r_{x}^{2}}} d x & \forall \zeta \in H_{p e r}^{1}(I),
\end{array}
$$

where $H_{p e r}^{1}(I)=\left\{\eta \in H^{1}(I) \mid \eta(0)=\eta(L)\right\}$. We employ (2.2), (2.3) in order to define a semidiscrete scheme using linear finite elements to approximate $r$ and $\kappa$. Let $0=x_{0}<x_{1}<\cdots<x_{N}=L, h_{j}:=x_{j}-x_{j-1}$, and $h:=\max _{1 \leq j \leq N} h_{j}$. We shall make an inverse assumption of the form

$$
h \leq \rho h_{j} \quad \forall j=1, \ldots, N,
$$

where $\rho>0$ is independent of $h$. The space of linear finite elements is defined by

$$
X_{h}:=\left\{\phi_{h} \in C^{0}(\bar{I}) \mid \phi_{h \mid\left[x_{j-1}, x_{j}\right]} \in P^{1}, 1 \leq j \leq N, \phi_{h}(0)=\phi_{h}(L)\right\} .
$$

Our discrete problem now reads as follows: find $r_{h}, \kappa_{h}:[0, T] \rightarrow X_{h}$ such that

$$
\begin{aligned}
\int_{I} r_{h} r_{h, t} \eta_{h} d x & =-\int_{I} \frac{r_{h} \kappa_{h, x} \eta_{h, x}}{\sqrt{1+r_{h, x}^{2}}} d x & & \forall \eta_{h} \in X_{h}, t \in(0, T], \\
\int_{I} r_{h} \kappa_{h} \zeta_{h} d x & =\int_{I} \sqrt{1+r_{h, x}^{2}} \zeta_{h} d x+\int_{I} \frac{r_{h} r_{h, x} \zeta_{h, x}}{\sqrt{1+r_{h, x}^{2}}} d x & & \forall \zeta_{h} \in X_{h}, t \in[0, T], \\
r_{h}(0) & =I_{h} r_{0}, & &
\end{aligned}
$$

where $I_{h}$ denotes the Lagrange interpolation operator.

LEMma 2.1. There exists $T_{h}>0$ such that (2.5)-(2.7) has a unique solution $\left(r_{h}, \kappa_{h}\right) \in C^{1}\left(\left[0, T_{h}\right] ; X_{h} \times X_{h}\right)$ satisfying $\frac{1}{2} \min _{[0, L]} r_{0} \leq r_{h} \leq 2 \max _{[0, L]} r_{0}$ in $[0, L] \times$ $\left[0, T_{h}\right]$.

Proof. Choose a smooth globally Lipschitz-continuous function $\beta: \mathbb{R} \rightarrow \mathbb{R}$ with the properties $\beta(s)=s$ for $\frac{1}{2} \min _{[0, L]} r_{0} \leq s \leq 2 \max _{[0, L]} r_{0}, \frac{1}{4} \min _{[0, L]} r_{0} \leq \beta(s) \leq$ $4 \max _{[0, L]} r_{0}$ for all $s \in \mathbb{R}$. We first consider the following modified problem: find $r_{h}, \kappa_{h}:[0, T] \rightarrow X_{h}$ such that

$$
\begin{array}{ll}
\int_{I} \beta\left(r_{h}\right) r_{h, t} \eta_{h} d x=-\int_{I} \frac{r_{h} \kappa_{h, x} \eta_{h, x}}{\sqrt{1+r_{h, x}^{2}}} d x & \forall \eta_{h} \in X_{h}, t \in[0, T] \\
\int_{I} \beta\left(r_{h}\right) \kappa_{h} \zeta_{h} d x=\int_{I} \sqrt{1+r_{h, x}^{2}} \zeta_{h} d x+\int_{I} \frac{r_{h} r_{h, x} \zeta_{h, x}}{\sqrt{1+r_{h, x}^{2}}} d x & \forall \zeta_{h} \in X_{h}, t \in[0, T]
\end{array}
$$

Denoting by $\psi_{1}, \ldots, \psi_{N}$ the usual nodal basis of $X_{h}$, we can represent $\left(r_{h}, \kappa_{h}\right)$ as

$$
r_{h}(\cdot, t)=\sum_{j=1}^{N} r_{j}(t) \psi_{j}, \quad \kappa_{h}(\cdot, t)=\sum_{j=1}^{N} \kappa_{j}(t) \psi_{j}
$$


and write $\underline{r}(t)=\left(r_{1}(t), \ldots, r_{N}(t)\right)^{T}, \underline{\kappa}(t)=\left(\kappa_{1}(t), \ldots, \kappa_{N}(t)\right)^{T}$. In view of the properties of $\beta$ we may rewrite (2.9) in the form $\underline{\kappa}(t)=G(\underline{r}(t))$ with a Lipschitz-continuous mapping $G: \mathbb{R}^{N} \rightarrow \mathbb{R}^{N}$. Inserting this into (2.8) and using again the properties of $\beta$, we may write this relation as

$$
\underline{r}^{\prime}(t)=F(\underline{r}(t)), \quad \underline{r}(0)=\left(r_{0}\left(x_{1}\right), \ldots, r_{0}\left(x_{N}\right)\right)^{T},
$$

with a Lipschitz-continuous $F: \mathbb{R}^{N} \rightarrow \mathbb{R}^{N}$. The existence and uniqueness of $\underline{r}$ on some interval $\left[0, T_{h}\right]$ follows directly from the theory of ODEs. The corresponding functions $r_{h}$ and $\kappa_{h}$ given by (2.11) will then solve (2.8)-(2.10). Since $r_{h}(0)=I_{h} r_{0}$ and by making $T_{h}$ smaller if necessary, we may assume that $\frac{1}{2} \min _{[0, L]} r_{0} \leq r_{h} \leq$ $2 \max _{[0, L]} r_{0}$ in $[0, L] \times\left[0, T_{h}\right]$ so that, in view of the properties of $\beta,\left(r_{h}, \kappa_{h}\right)$ also solves $(2.5)-(2.7)$.

Using $\eta_{h}=\kappa_{h}$ in (2.5) and $\zeta_{h}=r_{h, t}$ in (2.6) and taking the difference of the resulting equations, we obtain

$$
\begin{aligned}
0 & =\int_{I} \sqrt{1+r_{h, x}^{2}} r_{h, t} d x+\int_{I} \frac{r_{h} r_{h, x} r_{h, t x}}{\sqrt{1+r_{h, x}^{2}}} d x+\int_{I} \frac{r_{h} \kappa_{h, x}^{2}}{\sqrt{1+r_{h, x}^{2}}} d x \\
& =\frac{d}{d t} \int_{I} r_{h} \sqrt{1+r_{h, x}^{2}} d x+\int_{I} \frac{r_{h} \kappa_{h, x}^{2}}{\sqrt{1+r_{h, x}^{2}}} d x
\end{aligned}
$$

Thus

$$
\sup _{0 \leq t \leq T_{h}} \int_{I} r_{h} \sqrt{1+r_{h, x}^{2}} d x+\int_{0}^{T_{h}} \int_{I} \frac{r_{h} \kappa_{h, x}^{2}}{\sqrt{1+r_{h, x}^{2}}} d x d t \leq C\left(r_{0}\right)
$$

Before we formulate an error estimate for the scheme (2.5)-(2.7), we state a local existence and uniqueness result for the continuous problem.

THEOREM 2.2. Suppose that $r_{0} \in H_{\text {per }}^{4}(I)$ is strictly positive. Then there exists $T_{0}>0$ such that (1.3)-(1.6) has a unique solution $(r, \kappa)$, which satisfies $r \in$ $L^{\infty}\left(0, T_{0} ; H_{p e r}^{4}(I)\right), r_{t} \in L^{2}\left(0, T_{0} ; H_{p e r}^{2}(I)\right)$, and $r(x, t)>0$ for all $(x, t) \in I \times\left[0, T_{0}\right]$.

Proof. A similar result was proved in [11] for a formulation of (1.1) in terms of the distance function to a fixed reference curve. Since the resulting equation has the same structure as (1.3)-(1.6), the methods employed in [11] can be applied to our situation.

We denote by $\left[0, T_{\max }\right), T_{\max } \in(0, \infty]$ the maximal time interval on which the solution from Theorem 2.2 exists and fix $T<T_{\max }$. Then there exist constants $0<c_{0} \leq C_{0}$ and $M \geq 0$ (depending on $T$ ) such that

$$
\begin{gathered}
c_{0} \leq r \leq C_{0}, \quad\left|r_{x}\right| \leq C_{0} \quad \text { on }[0, L] \times[0, T], \\
\sup _{t \in(0, T)}\|r(., t)\|_{H^{4}(I)}^{2}+\int_{0}^{T}\left\|r_{t}\right\|_{H^{2}(I)}^{2} d t \leq M^{2} .
\end{gathered}
$$

Combining these bounds with (1.2), (1.3), and the inequality $\|f\|_{L^{\infty}(I)} \leq C\|f\|_{H^{1}(I)}$, 
we note for later use

$$
\|\kappa(., t)\|_{H^{1, \infty}(I)}+\|\kappa(., t)\|_{H^{2}(I)}+\left\|r_{t}(., t)\right\|_{L^{2}(I)} \leq C \quad \text { uniformly in } t \in[0, T],
$$

where $C$ depends on $L, c_{0}, C_{0}$, and $M$.

Our main result is the following error estimate, the proof of which will be given in the next section.

TheOrem 2.3. There exists an $h_{0}>0$ such that for all $0<h \leq h_{0}$ the discrete solution $\left(r_{h}, \kappa_{h}\right)$ exists on $[0, T]$ and

$$
\sup _{0 \leq t \leq T}\left\|\left(r-r_{h}\right)(t)\right\|_{H^{1}(I)}^{2}+\int_{0}^{T}\left\|\kappa-\kappa_{h}\right\|_{H^{1}(I)}^{2} d t \leq C h^{2} .
$$

The constant $C$ depends on $L, T, c_{0}, C_{0}, M$, and $\rho$.

3. Proof of Theorem 2.3. Let us define

$$
\begin{gathered}
\hat{T}_{h}:=\sup \left\{t \in[0, T] \mid\left(r_{h}, \kappa_{h}\right) \text { solves }(2.5)-(2.7) \text { on }[0, t]\right. \text { and } \\
\left.\frac{1}{2} c_{0} \leq r_{h} \leq 2 C_{0},\left|r_{h, x}\right| \leq 2 C_{0} \text { on }[0, t]\right\} .
\end{gathered}
$$

By choosing $T_{h}$ smaller if necessary (in order to satisfy the bound on $r_{h, x}$ ), we may deduce from Lemma 2.1 that $\hat{T}_{h}>0$. Our aim is to show that $\hat{T}_{h}=T$ for small $h$. This will be achieved by proving the bounds $(2.16)$ on $\left[0, \hat{T}_{h}\right]$, which will enable us to continue the discrete solution. By the definition of $\hat{T}_{h}$ we have

$$
\frac{1}{2} c_{0} \leq r_{h} \leq 2 C_{0}, \quad\left|r_{h, x}\right| \leq 2 C_{0} \text { on }[0, L] \times\left[0, \hat{T}_{h}\right) .
$$

In what follows, we shall denote by $C$ a constant which may depend on $L, T, c_{0}, C_{0}, M$, and $\rho$. Additional dependencies of $C$ will be stated explicitly. We start with a useful auxiliary lemma.

Lemma 3.1. Let $v \in H_{p e r}^{1}(I), t \in\left[0, \hat{T}_{h}\right)$. Then we have for $\epsilon>0$

$$
\begin{aligned}
\left|\int_{I} \frac{r_{h}}{r} v r r_{t} d x-\int_{I} v r_{h} r_{h, t} d x\right| \leq \epsilon\left\|\kappa_{x}-\kappa_{h, x}\right\|_{L^{2}(I)}^{2} & +C_{\epsilon}\|v\|_{H^{1}(I)}^{2} \\
& +C h^{2}+C\left\|r-r_{h}\right\|_{H^{1}(I)}^{2} .
\end{aligned}
$$

Proof. Fix $t \in\left[0, \hat{T}_{h}\right)$ and denote by $Q_{h}: L^{2}(I) \rightarrow X_{h}$ the following weighted projection: for a given $u \in L^{2}(I)$ let $Q_{h} u \in X_{h}$ be defined by

$$
\int_{I} r_{h} u \zeta_{h} d x=\int_{I} r_{h} Q_{h} u \zeta_{h} d x \quad \forall \zeta_{h} \in X_{h}
$$

We claim that

$$
\left\|u-Q_{h} u\right\|_{L^{2}(I)}+h\left\|u_{x}-\left(Q_{h} u\right)_{x}\right\|_{L^{2}(I)} \leq C h\left\|u_{x}\right\|_{L^{2}(I)} \quad \forall u \in H_{p e r}^{1}(I) .
$$

To see this, we first note that (3.1), (3.2), and an interpolation inequality imply

$$
\begin{aligned}
\frac{c_{0}}{2} \int_{I}\left|u-Q_{h} u\right|^{2} & \leq \int_{I} r_{h}\left(u-Q_{h} u\right)\left(u-Q_{h} u\right)=\int_{I} r_{h}\left(u-Q_{h} u\right)\left(u-I_{h} u\right) \\
& \leq 2 C_{0}\left\|u-Q_{h} u\right\|_{L^{2}(I)} h\left\|u_{x}\right\|_{L^{2}(I)},
\end{aligned}
$$


which yields the first part of (3.3). In view of (2.4) we have that $\left\|v_{h, x}\right\|_{L^{2}(I)} \leq$ $C h^{-1}\left\|v_{h}\right\|_{L^{2}(I)}$ for $v_{h} \in X_{h}$, and therefore

$$
\begin{aligned}
\left\|u_{x}-\left(Q_{h} u\right)_{x}\right\|_{L^{2}(I)} & \leq\left\|u_{x}-\left(I_{h} u\right)_{x}\right\|_{L^{2}(I)}+\left\|\left(I_{h} u\right)_{x}-\left(Q_{h} u\right)_{x}\right\|_{L^{2}(I)} \\
& \leq 2\left\|u_{x}\right\|_{L^{2}(I)}+C h^{-1}\left\|I_{h} u-Q_{h} u\right\|_{L^{2}(I)} \\
& \leq 2\left\|u_{x}\right\|_{L^{2}(I)}+C h^{-1}\left(\left\|u-I_{h} u\right\|_{L^{2}(I)}+\left\|u-Q_{h} u\right\|_{L^{2}(I)}\right) \\
& \leq C\left\|u_{x}\right\|_{L^{2}(I)}
\end{aligned}
$$

where we used the bound on $\left\|u-Q_{u}\right\|_{L^{2}(I)}$. This proves (3.3).

Next we infer from (3.2) and (2.5) that

$$
\int_{I} v r_{h} r_{h, t} d x=\int_{I} Q_{h} v r_{h} r_{h, t} d x=-\int_{I} \frac{r_{h} \kappa_{h, x}\left(Q_{h} v\right)_{x}}{\sqrt{1+r_{h, x}^{2}}} d x \quad \forall v \in H_{p e r}^{1}(I) .
$$

If we combine this relation with (2.2), we may continue with

$$
\begin{aligned}
& \int_{I} \frac{r_{h}}{r} v r r_{t} d x-\int_{I} v r_{h} r_{h, t} d x \\
& =-\int_{I} \frac{r \kappa_{x}\left(\frac{r_{h}}{r} v\right)_{x}}{\sqrt{1+r_{x}^{2}}} d x+\int_{I} \frac{r_{h} \kappa_{h, x}\left(Q_{h} v\right)_{x}}{\sqrt{1+r_{h, x}^{2}}} d x \\
& =-\int_{I} \frac{r \kappa_{x} v}{\sqrt{1+r_{x}^{2}}} \frac{r_{h, x} r-r_{x} r_{h}}{r^{2}} d x+\int_{I} \frac{r-r_{h}}{\sqrt{1+r_{x}^{2}}} \kappa_{x} v_{x} d x \\
& \quad+\int_{I}\left(\frac{r_{h}}{\sqrt{1+r_{h, x}^{2}}}-\frac{r}{\sqrt{1+r_{x}^{2}}}\right) \kappa_{h, x}\left(Q_{h} v\right)_{x} d x \\
& \quad+\int_{I} \frac{r}{\sqrt{1+r_{x}^{2}}}\left(\kappa_{h, x}-\kappa_{x}\right)\left(Q_{h} v\right)_{x} d x+\int_{I} \frac{r \kappa_{x}}{\sqrt{1+r_{x}^{2}}}\left(Q_{h} v-v\right)_{x} d x \\
& \equiv \sum_{i=1}^{5} S_{i} .
\end{aligned}
$$

In view of (2.13), (2.15), and (3.1), we then have

$$
\begin{aligned}
& \left|S_{1}\right| \leq C \int_{I}|v|\left(\left|r-r_{h}\right|+\left|r_{x}-r_{h, x}\right|\right) d x \leq\|v\|_{L^{2}(I)}^{2}+C\left\|r-r_{h}\right\|_{H^{1}(I)}^{2}, \\
& \left|S_{2}\right| \leq\left\|v_{x}\right\|_{L^{2}(I)}^{2}+C\left\|r-r_{h}\right\|_{L^{2}(I)}^{2} .
\end{aligned}
$$

Next, (2.15) and (3.3) imply

$$
\begin{aligned}
\left|S_{3}\right| & \leq \int_{I}\left|\frac{r_{h}}{\sqrt{1+r_{h, x}^{2}}}-\frac{r}{\sqrt{1+r_{x}^{2}}}\right|\left(\left|\kappa_{x}\right|+\left|\kappa_{h, x}-\kappa_{x}\right|\right)\left|\left(Q_{h} v\right)_{x}\right| d x \\
& \leq C \int_{I}\left(\left|r-r_{h}\right|+\left|r_{x}-r_{h, x}\right|\right)\left|\left(Q_{h} v\right)_{x}\right| d x+C \int_{I}\left|\kappa_{x}-\kappa_{h, x}\right|\left|\left(Q_{h} v\right)_{x}\right| d x \\
& \leq \epsilon\left\|\kappa_{x}-\kappa_{h, x}\right\|_{L^{2}(I)}^{2}+C_{\epsilon}\left\|v_{x}\right\|_{L^{2}(I)}^{2}+C\left\|r-r_{h}\right\|_{H^{1}(I)}^{2},
\end{aligned}
$$

and similarly,

$$
\left|S_{4}\right| \leq \epsilon\left\|\kappa_{x}-\kappa_{h, x}\right\|_{L^{2}(I)}^{2}+C_{\epsilon}\left\|v_{x}\right\|_{L^{2}(I)}^{2}
$$


Finally, integration by parts, (1.3), (2.15), and (3.3) yield

$$
\begin{aligned}
\left|S_{5}\right| & =\left|-\int_{I}\left(\frac{r \kappa_{x}}{\sqrt{1+r_{x}^{2}}}\right)_{x}\left(Q_{h} v-v\right) d x\right| \\
& \leq C h\left\|v_{x}\right\|_{L^{2}(I)}\left\|r_{t}\right\|_{L^{2}(I)} \leq C h^{2}+C\left\|v_{x}\right\|_{L^{2}(I)}^{2} .
\end{aligned}
$$

Collecting the above estimates concludes the proof of the lemma.

As a first application of the above result we derive a differential inequality for the $L^{2}$-error.

LEMMA 3.2 .

$$
\frac{1}{2} \frac{d}{d t}\left\|r-r_{h}\right\|_{L^{2}(I)}^{2} \leq \epsilon\left\|\kappa_{x}-\kappa_{h, x}\right\|_{L^{2}(I)}^{2}+C_{\epsilon}\left\|r-r_{h}\right\|_{H^{1}(I)}^{2}+C h^{2} .
$$

Proof. Clearly,

$$
\begin{aligned}
\frac{1}{2} \frac{d}{d t}\left\|r-r_{h}\right\|_{L^{2}(I)}^{2} & =\int_{I}\left(r-r_{h}\right)\left(r_{t}-r_{h, t}\right) d x \\
& =\int_{I} \frac{1}{r}\left(r-r_{h}\right) r r_{t} d x-\int_{I} \frac{1}{r_{h}}\left(r-r_{h}\right) r_{h} r_{h, t} d x .
\end{aligned}
$$

If we apply Lemma 3.1 to the function

$$
v:=\frac{1}{r_{h}}\left(r-r_{h}\right)(\cdot, t) \quad \text { for } t \in\left(0, \hat{T}_{h}\right),
$$

the result follows.

The main part of the proof of Theorem 2.3 consists in controlling the $H^{1}$ seminorms of $r-r_{h}$ and $\kappa-\kappa_{h}$. The idea is to mimic the argument which led to the a priori estimate (2.12) in such a way that it can be applied to the difference between exact and discrete solution. This suggests using $\eta_{h}=I_{h} \kappa-\kappa_{h}, \zeta_{h}=I_{h} r_{t}-r_{h, t}$ in the error relations satisfied by $r-r_{h}, \kappa-\kappa_{h}$. In order to derive these relations we use $\eta=\eta_{h} \in X_{h}$ in (2.2) and $\zeta=\zeta_{h} \in X_{h}$ in (2.3) and take the difference with (2.5), (2.6), respectively. This leads to

$$
\begin{aligned}
\int_{I}\left(r r_{t}-r_{h} r_{h, t}\right) \eta_{h} d x= & -\int_{I}\left(\frac{r \kappa_{x}}{\sqrt{1+r_{x}^{2}}}-\frac{r_{h} \kappa_{h, x}}{\sqrt{1+r_{h, x}^{2}}}\right) \eta_{h, x} d x \quad \forall \eta_{h} \in X_{h}, \\
\int_{I}\left(r \kappa-r_{h} \kappa_{h}\right) \zeta_{h} d x= & \int_{I}\left(\sqrt{1+r_{x}^{2}}-\sqrt{1+r_{h, x}^{2}}\right) \zeta_{h} d x \\
& +\int_{I}\left(\frac{r r_{x}}{\sqrt{1+r_{x}^{2}}}-\frac{r_{h} r_{h, x}}{\sqrt{1+r_{h, x}^{2}}}\right) \zeta_{h, x} d x \quad \forall \zeta_{h} \in X_{h} .
\end{aligned}
$$

Lemma 3.3. We have for all $\epsilon>0$

$$
\begin{aligned}
& \frac{d}{d t} \int_{I} r_{h}\left(\sqrt{1+r_{h, x}^{2}}-\frac{r_{h, x} r_{x}+1}{\sqrt{1+r_{x}^{2}}}\right) d x+\int_{I} \frac{r_{h}}{\sqrt{1+r_{h, x}^{2}}}\left(\kappa_{x}-\kappa_{h, x}\right)^{2} d x \\
& \leq C \epsilon\left\|\kappa-\kappa_{h}\right\|_{H^{1}(I)}^{2}+C_{\epsilon}\left(1+\left\|r_{t}\right\|_{H^{2}(I)}\right)\left\|r-r_{h}\right\|_{H^{1}(I)}^{2}+C_{\epsilon} h^{2}\left(1+\left\|r_{t}\right\|_{H^{2}(I)}^{2}\right) .
\end{aligned}
$$


Proof. Using $\zeta_{h}=I_{h} r_{t}-r_{h, t}$ in (3.6), we obtain

$$
\begin{aligned}
\int_{I}(r \kappa- & \left.r_{h} \kappa_{h}\right)\left(I_{h} r_{t}-r_{h, t}\right) d x=\int_{I}\left(\sqrt{1+r_{x}^{2}}-\sqrt{1+r_{h, x}^{2}}\right)\left(I_{h} r_{t}-r_{h, t}\right) d x \\
& +\int_{I} r_{h}\left(\frac{r_{x}}{\sqrt{1+r_{x}^{2}}}-\frac{r_{h, x}}{\sqrt{1+r_{h, x}^{2}}}\right)\left(r_{t x}-r_{h, t x}\right) d x \\
& +\int_{I}\left(r-r_{h}\right) \frac{r_{x}}{\sqrt{1+r_{x}^{2}}}\left(r_{t x}-r_{h, t x}\right) d x \\
& +\int_{I}\left(\frac{r r_{x}}{\sqrt{1+r_{x}^{2}}}-\frac{r_{h} r_{h, x}}{\sqrt{1+r_{h, x}^{2}}}\right)\left(\left(I_{h} r_{t}\right)_{x}-r_{t x}\right) d x .
\end{aligned}
$$

Note first that the second integral can be written as

$$
\begin{aligned}
& \int_{I} r_{h}\left(\frac{r_{x}}{\sqrt{1+r_{x}^{2}}}-\frac{r_{h, x}}{\sqrt{1+r_{h, x}^{2}}}\right)\left(r_{t x}-r_{h, t x}\right) d x \\
& =\int_{I} r_{h} \frac{\partial}{\partial t}\left(\sqrt{1+r_{h, x}^{2}}-\frac{r_{h, x} r_{x}+1}{\sqrt{1+r_{x}^{2}}}\right) d x \\
& \quad+\int_{I} r_{h} r_{t, x}\left(\frac{r_{h, x}}{\sqrt{1+r_{x}^{2}}}-\frac{r_{h, x}}{\sqrt{1+r_{h, x}^{2}}}+\frac{r_{x}}{\sqrt{1+r_{x}^{2}}}-\frac{1+r_{x} r_{h, x}}{1+r_{x}^{2}} \frac{r_{x}}{\sqrt{1+r_{x}^{2}}}\right) d x \\
& =\frac{d}{d t} \int_{I} r_{h}\left(\sqrt{1+r_{h, x}^{2}}-\frac{r_{h, x} r_{x}+1}{\sqrt{1+r_{x}^{2}}}\right) d x-\int_{I} r_{h, t}\left(\sqrt{1+r_{h, x}^{2}}-\frac{r_{h, x} r_{x}+1}{\sqrt{1+r_{x}^{2}}}\right) d x \\
& \quad+\int_{I} r_{h} r_{t, x}\left(\frac{r_{h, x}}{\sqrt{1+r_{x}^{2}}}-\frac{r_{h, x}}{\sqrt{1+r_{h, x}^{2}}}+\frac{r_{x}}{\sqrt{1+r_{x}^{2}}}-\frac{1+r_{x} r_{h, x}}{1+r_{x}^{2}} \frac{r_{x}}{\sqrt{1+r_{x}^{2}}}\right) d x
\end{aligned}
$$

Integration by parts together with (1.2) implies for the third term in (3.8)

$$
\begin{aligned}
& \int_{I}\left(r-r_{h}\right) \frac{r_{x}}{\sqrt{1+r_{x}^{2}}}\left(r_{t x}-r_{h, t x}\right) d x \\
& =-\int_{I}\left(r_{x}-r_{h, x}\right) \frac{r_{x}}{\sqrt{1+r_{x}^{2}}}\left(r_{t}-r_{h, t}\right) d x-\int_{I}\left(r-r_{h}\right)\left(\frac{r_{x}}{\sqrt{1+r_{x}^{2}}}\right)_{x}\left(r_{t}-r_{h, t}\right) d x \\
& =-\int_{I} r_{t}\left(r_{x}-r_{h, x}\right) \frac{r_{x}}{\sqrt{1+r_{x}^{2}}} d x+\int_{I} r_{h, t} \sqrt{1+r_{x}^{2}} d x-\int_{I} r_{h, t} \frac{r_{h, x} r_{x}+1}{\sqrt{1+r_{x}^{2}}} d x \\
& \quad-\int_{I}\left(r-r_{h}\right) \frac{1}{r \sqrt{1+r_{x}^{2}}}\left(r_{t}-r_{h, t}\right) d x+\int_{I}\left(r-r_{h}\right) \kappa\left(r_{t}-r_{h, t}\right) d x
\end{aligned}
$$

Inserting the above equations into (3.8), we derive

$$
\begin{gathered}
\int_{I}\left(r \kappa-r_{h} \kappa_{h}\right)\left(I_{h} r_{t}-r_{h, t}\right) d x=\frac{d}{d t} \int_{I} r_{h}\left(\sqrt{1+r_{h, x}^{2}}-\frac{r_{h, x} r_{x}+1}{\sqrt{1+r_{x}^{2}}}\right) d x \\
\quad+\int_{I}\left(\sqrt{1+r_{x}^{2}}-\sqrt{1+r_{h, x}^{2}}\right) I_{h} r_{t} d x-\int_{I} r_{t}\left(r_{x}-r_{h, x}\right) \frac{r_{x}}{\sqrt{1+r_{x}^{2}}} d x
\end{gathered}
$$




$$
\begin{aligned}
& +\int_{I} r_{h} r_{t x}\left(\frac{r_{h, x}}{\sqrt{1+r_{x}^{2}}}-\frac{r_{h, x}}{\sqrt{1+r_{h, x}^{2}}}+\frac{r_{x}}{\sqrt{1+r_{x}^{2}}}-\frac{1+r_{x} r_{h, x}}{1+r_{x}^{2}} \frac{r_{x}}{\sqrt{1+r_{x}^{2}}}\right) d x \\
& -\int_{I}\left(r-r_{h}\right) \frac{1}{r \sqrt{1+r_{x}^{2}}}\left(r_{t}-r_{h, t}\right) d x+\int_{I}\left(r-r_{h}\right) \kappa\left(r_{t}-r_{h, t}\right) d x \\
& +\int_{I}\left(\frac{r r_{x}}{\sqrt{1+r_{x}^{2}}}-\frac{r_{h} r_{h, x}}{\sqrt{1+r_{h, x}^{2}}}\right)\left(\left(I_{h} r_{t}\right)_{x}-r_{t x}\right) d x .
\end{aligned}
$$

Let us next insert $\eta_{h}=I_{h} \kappa-\kappa_{h}$ into (3.5):

$$
\begin{aligned}
\int_{I}\left(r r_{t}\right. & \left.-r_{h} r_{h, t}\right)\left(I_{h} \kappa-\kappa_{h}\right) d x \\
= & -\int_{I}\left(\frac{r \kappa_{x}}{\sqrt{1+r_{x}^{2}}}-\frac{r_{h} \kappa_{h, x}}{\sqrt{1+r_{h, x}^{2}}}\right)\left(\left(I_{h} \kappa\right)_{x}-\kappa_{h, x}\right) d x \\
= & \int_{I}\left(\frac{r \kappa_{x}}{\sqrt{1+r_{x}^{2}}}-\frac{r_{h} \kappa_{h, x}}{\sqrt{1+r_{h, x}^{2}}}\right)\left(\kappa_{x}-\left(I_{h} \kappa\right)_{x}\right) d x \\
& -\int_{I} \frac{r_{h}}{\sqrt{1+r_{h, x}^{2}}}\left(\kappa_{x}-\kappa_{h, x}\right)^{2} d x \\
& -\int_{I}\left(\frac{r}{\sqrt{1+r_{x}^{2}}}-\frac{r_{h}}{\sqrt{1+r_{h, x}^{2}}}\right) \kappa_{x}\left(\kappa_{x}-\kappa_{h, x}\right) d x .
\end{aligned}
$$

Combining (3.9) and (3.10), we obtain

$$
\frac{d}{d t} \int_{I} r_{h}\left(\sqrt{1+r_{h, x}^{2}}-\frac{r_{h, x} r_{x}+1}{\sqrt{1+r_{x}^{2}}}\right) d x+\int_{I} \frac{r_{h}}{\sqrt{1+r_{h, x}^{2}}}\left(\kappa_{x}-\kappa_{h, x}\right)^{2} d x=\sum_{i=1}^{8} \tilde{S}_{i}
$$

where

$$
\begin{aligned}
\tilde{S}_{1}= & \int_{I}\left(r \kappa-r_{h} \kappa_{h}\right)\left(I_{h} r_{t}-r_{h, t}\right) d x-\int_{I}\left(r r_{t}-r_{h} r_{h, t}\right)\left(I_{h} \kappa-\kappa_{h}\right) d x \\
& -\int_{I}\left(r-r_{h}\right) \kappa\left(r_{t}-r_{h, t}\right) d x \\
\tilde{S}_{2}= & -\int_{I} r_{t}\left(\sqrt{1+r_{x}^{2}}-\sqrt{1+r_{h, x}^{2}}-\left(r_{x}-r_{h, x}\right) \frac{r_{x}}{\sqrt{1+r_{x}^{2}}}\right) d x \\
\tilde{S}_{3}= & -\int_{I} r_{h} r_{t x}\left(\frac{r_{h, x}}{\sqrt{1+r_{x}^{2}}}-\frac{r_{h, x}}{\sqrt{1+r_{h, x}^{2}}}+\frac{r_{x}}{\sqrt{1+r_{x}^{2}}}-\frac{1+r_{x} r_{h, x}}{1+r_{x}^{2}} \frac{r_{x}}{\sqrt{1+r_{x}^{2}}}\right) d x \\
\tilde{S}_{4}= & \int_{I}\left(r-r_{h}\right) \frac{1}{r \sqrt{1+r_{x}^{2}}}\left(r_{t}-r_{h, t}\right) d x \\
\tilde{S}_{5}= & \int_{I}\left(\sqrt{1+r_{x}^{2}}-\sqrt{1+r_{h, x}^{2}}\right)\left(r_{t}-I_{h} r_{t}\right) d x
\end{aligned}
$$




$$
\begin{aligned}
& \tilde{S}_{6}=-\int_{I}\left(\frac{r r_{x}}{\sqrt{1+r_{x}^{2}}}-\frac{r_{h} r_{h, x}}{\sqrt{1+r_{h, x}^{2}}}\right)\left(\left(I_{h} r_{t}\right)_{x}-r_{t x}\right) d x, \\
& \tilde{S}_{7}=\int_{I}\left(\frac{r \kappa_{x}}{\sqrt{1+r_{x}^{2}}}-\frac{r_{h} \kappa_{h, x}}{\sqrt{1+r_{h, x}^{2}}}\right)\left(\kappa_{x}-\left(I_{h} \kappa\right)_{x}\right) d x, \\
& \tilde{S}_{8}=-\int_{I}\left(\frac{r}{\sqrt{1+r_{x}^{2}}}-\frac{r_{h}}{\sqrt{1+r_{h, x}^{2}}}\right) \kappa_{x}\left(\kappa_{x}-\kappa_{h, x}\right) d x .
\end{aligned}
$$

The terms $\tilde{S}_{1}, \ldots, \tilde{S}_{8}$ have been organized in such a way that each of them is quadratic in an appropriate difference. To see this, let us examine them in more detail. First,

$$
\begin{aligned}
\tilde{S}_{1} & =\int_{I}\left(r \kappa-r_{h} \kappa_{h}\right)\left(r_{t}-r_{h, t}\right) d x-\int_{I}\left(r r_{t}-r_{h} r_{h, t}\right)\left(\kappa-\kappa_{h}\right) d x-\int_{I}\left(r-r_{h}\right) \kappa\left(r_{t}-r_{h, t}\right) d x \\
& +\int_{I}\left(r \kappa-r_{h} \kappa_{h}\right)\left(I_{h} r_{t}-r_{t}\right) d x-\int_{I}\left(r r_{t}-r_{h} r_{h, t}\right)\left(I_{h} \kappa-\kappa\right) d x \\
= & -\int_{I} r_{t}\left(\kappa-\kappa_{h}\right)\left(r-r_{h}\right) d x+\int_{I}\left(r \kappa-r_{h} \kappa_{h}\right)\left(I_{h} r_{t}-r_{t}\right) d x-\int_{I}\left(r r_{t}-r_{h} r_{h, t}\right)\left(I_{h} \kappa-\kappa\right) d x \\
\equiv & A_{1}+A_{2}+A_{3} .
\end{aligned}
$$

Using an interpolation estimate, (2.15), and the continuous embedding $H^{1}(I) \hookrightarrow$ $L^{\infty}(I)$, we obtain

$$
\begin{aligned}
\left|A_{1}+A_{2}\right| & \leq C\left\|r_{t}\right\|_{L^{2}(I)}\left\|\kappa-\kappa_{h}\right\|_{L^{2}(I)}\left\|r-r_{h}\right\|_{L^{\infty}(I)}+C h\left\|r_{t x}\right\|_{L^{2}(I)}\left\|r \kappa-r_{h} \kappa_{h}\right\|_{L^{2}(I)} \\
& \leq \epsilon\left\|\kappa-\kappa_{h}\right\|_{L^{2}(I)}^{2}+C_{\epsilon}\left\|r-r_{h}\right\|_{H^{1}(I)}^{2}+C_{\epsilon} h^{2}\left\|r_{t x}\right\|_{L^{2}(I)}^{2},
\end{aligned}
$$

while

$$
A_{3}=\int_{I}\left(I_{h} \kappa-\kappa\right) r_{h} r_{h, t} d x-\int_{I} \frac{r_{h}}{r}\left(I_{h} \kappa-\kappa\right) r r_{t} d x+\int_{I}\left(I_{h} \kappa-\kappa\right)\left(\frac{r_{h}}{r}-1\right) r r_{t} d x
$$

We infer from Lemma 3.1 with $v=\kappa-I_{h} \kappa$ and well-known interpolation estimates that

$$
\begin{gathered}
\left|A_{3}\right| \leq \epsilon\left\|\kappa_{x}-\kappa_{h, x}\right\|_{L^{2}(I)}^{2}+C_{\epsilon}\left\|\kappa-I_{h} \kappa\right\|_{H^{1}(I)}^{2}+C h^{2}+C\left\|r-r_{h}\right\|_{H^{1}(I)}^{2} \\
\quad+C\left\|r_{t}\right\|_{L^{2}(I)}\left\|r-r_{h}\right\|_{L^{\infty}(I)}\left\|\kappa-I_{h} \kappa\right\|_{L^{2}(I)} \\
\leq \epsilon\left\|\kappa_{x}-\kappa_{h, x}\right\|_{L^{2}(I)}^{2}+C_{\epsilon} h^{2}\|\kappa\|_{H^{2}(I)}^{2}+C\left\|r-r_{h}\right\|_{H^{1}(I)}^{2} .
\end{gathered}
$$

Recalling (2.15), we conclude

$$
\left|\tilde{S}_{1}\right| \leq \epsilon\left\|\kappa-\kappa_{h}\right\|_{H^{1}(I)}^{2}+C_{\epsilon}\left\|r-r_{h}\right\|_{H^{1}(I)}^{2}+C_{\epsilon}\left(1+\left\|r_{t x}\right\|_{L^{2}(I)}^{2}\right) h^{2} .
$$

Next, observing that

$$
\left|\sqrt{1+q^{2}}-\sqrt{1+p^{2}}-(q-p) \frac{q}{\sqrt{1+q^{2}}}\right| \leq C(q-p)^{2} \quad \forall q, p \in \mathbb{R},
$$

we obtain

$$
\left|\tilde{S}_{2}\right| \leq C\left\|r_{t}\right\|_{L^{\infty}(I)}\left\|r_{x}-r_{h, x}\right\|_{L^{2}(I)}^{2} \leq C\left\|r_{t}\right\|_{H^{1}(I)}\left\|r_{x}-r_{h, x}\right\|_{L^{2}(I)}^{2} .
$$


Let us now examine $\tilde{S}_{3}$. A short calculation shows

$$
\begin{aligned}
& \frac{p}{\sqrt{1+q^{2}}}-\frac{p}{\sqrt{1+p^{2}}}+\frac{q}{\sqrt{1+q^{2}}}-\frac{1+p q}{1+q^{2}} \frac{q}{\sqrt{1+q^{2}}} \\
& =\frac{p\left(1+q^{2}\right)\left(\sqrt{1+p^{2}}-\sqrt{1+q^{2}}\right)-q^{2} \sqrt{1+p^{2}}(p-q)}{\sqrt{1+q^{2}}{ }^{3} \sqrt{1+p^{2}}} \\
& =\frac{p}{\sqrt{1+q^{2}} \sqrt{1+p^{2}}}\left(\sqrt{1+p^{2}}-\sqrt{1+q^{2}}-(p-q) \frac{p}{\sqrt{1+p^{2}}}\right) \\
& \quad+\frac{p-q}{{\sqrt{1+q^{2}}}^{3}\left(1+p^{2}\right)}\left(p^{2}\left(1+q^{2}\right)-q^{2}\left(1+p^{2}\right)\right),
\end{aligned}
$$

which implies in view of (3.12)

$$
\left|\frac{p}{\sqrt{1+q^{2}}}-\frac{p}{\sqrt{1+p^{2}}}+\frac{q}{\sqrt{1+q^{2}}}-\frac{1+p q}{1+q^{2}} \frac{q}{\sqrt{1+q^{2}}}\right| \leq C(p-q)^{2}
$$

for all $p, q \in \mathbb{R}$. Therefore,

$$
\left|\tilde{S}_{3}\right| \leq C\left\|r_{t x}\right\|_{L^{\infty}(I)}\left\|r_{x}-r_{h, x}\right\|_{L^{2}(I)}^{2} \leq C\left\|r_{t x}\right\|_{H^{1}(I)}\left\|r_{x}-r_{h, x}\right\|_{L^{2}(I)}^{2} .
$$

If we write

$$
\tilde{S}_{4}=\int_{I}\left(r-r_{h}\right) \frac{1}{r \sqrt{1+r_{x}^{2}}}\left(r_{t}-r_{h, t}\right) d x=\int_{I} \frac{r_{h}}{r} v r r_{t} d x-\int_{I} v r_{h} r_{h, t} d x
$$

with $v=\frac{r-r_{h}}{r_{h} r \sqrt{1+r_{x}^{2}}}$ and apply Lemma 3.1, we deduce

$$
\begin{aligned}
\left|\tilde{S}_{4}\right| & \leq \epsilon\left\|\kappa_{x}-\kappa_{h, x}\right\|_{L^{2}(I)}^{2}+C_{\epsilon}\left\|\frac{r-r_{h}}{r_{h} r \sqrt{1+r_{x}^{2}}}\right\|_{H^{1}(I)}^{2}+C h^{2}+C\left\|r-r_{h}\right\|_{H^{1}(I)}^{2} \\
& \leq \epsilon\left\|\kappa_{x}-\kappa_{h, x}\right\|_{L^{2}(I)}^{2}+C_{\epsilon}\left\|r-r_{h}\right\|_{H^{1}(I)}^{2}+C h^{2} .
\end{aligned}
$$

In view of interpolation estimates, Young's inequality, and (2.15),

$$
\begin{aligned}
\left|\tilde{S}_{5}\right| & \leq C h\left\|r_{t x}\right\|_{L^{2}(I)}\left\|r_{x}-r_{h, x}\right\|_{L^{2}(I)} \leq C h^{2}\left\|r_{t x}\right\|_{L^{2}(I)}^{2}+C\left\|r_{x}-r_{h, x}\right\|_{L^{2}(I)}^{2}, \\
\left|\tilde{S}_{6}\right| & \leq C h\left\|r_{t x x}\right\|_{L^{2}(I)}\left\|r-r_{h}\right\|_{H^{1}(I)} \leq C h^{2}\left\|r_{t x x}\right\|_{L^{2}(I)}^{2}+C\left\|r-r_{h}\right\|_{H^{1}(I)}^{2}, \\
\left|\tilde{S}_{7}\right| & \leq C h\left\|\kappa_{x x}\right\|_{L^{2}(I)}\left(\left\|\kappa_{x}-\kappa_{h, x}\right\|_{L^{2}(I)}+\left\|r-r_{h}\right\|_{H^{1}(I)}\right) \\
& \leq \epsilon\left\|\kappa_{x}-\kappa_{h, x}\right\|_{L^{2}(I)}^{2}+C_{\epsilon} h^{2}+C\left\|r-r_{h}\right\|_{H^{1}(I)}^{2} .
\end{aligned}
$$

Finally,

$$
\left|\tilde{S}_{8}\right| \leq \epsilon\left\|\kappa_{x}-\kappa_{h, x}\right\|_{L^{2}(I)}^{2}+C_{\epsilon}\left\|r-r_{h}\right\|_{H^{1}(I)}^{2} .
$$

If we insert the above estimates for $\tilde{S}_{1}, \ldots, \tilde{S}_{8}$ into (3.11), the result is

$$
\begin{aligned}
\frac{d}{d t} \int_{I} r_{h} & \left(\sqrt{1+r_{h, x}^{2}}-\frac{r_{h, x} r_{x}+1}{\sqrt{1+r_{x}^{2}}}\right) d x+\int_{I} \frac{r_{h}}{\sqrt{1+r_{h, x}^{2}}}\left(\kappa_{x}-\kappa_{h, x}\right)^{2} d x \\
& \leq C \epsilon\left\|\kappa-\kappa_{h}\right\|_{H^{1}(I)}^{2}+C_{\epsilon}\left(1+\left\|r_{t}\right\|_{H^{2}(I)}\right)\left\|r-r_{h}\right\|_{H^{1}(I)}^{2}+C_{\epsilon}\left(1+\left\|r_{t}\right\|_{H^{2}(I)}^{2}\right) h^{2},
\end{aligned}
$$

which completes the proof of the lemma. 
Remark 3.4. (a) In order to interpret the integral

$$
\int_{I} r_{h}\left(\sqrt{1+r_{h, x}^{2}}-\frac{r_{h, x} r_{x}+1}{\sqrt{1+r_{x}^{2}}}\right) d x
$$

occurring in (3.7), we note that

$$
\nu=\frac{1}{\sqrt{1+r_{x}^{2}}}\left(-r_{x}, \cos \phi, \sin \phi\right), \quad \nu_{h}=\frac{1}{\sqrt{1+r_{h, x}^{2}}}\left(-r_{h, x}, \cos \phi, \sin \phi\right)
$$

are the unit outward normals to

$$
\begin{aligned}
\Gamma(t) & =\left\{\mathbf{x} \in \mathbb{R}^{3} \mid \mathbf{x}=(x, r(x, t) \cos \phi, r(x, t) \sin \phi), x \in[0, L], \phi \in[0,2 \pi]\right\}, \\
\Gamma_{h}(t) & =\left\{\mathbf{x} \in \mathbb{R}^{3} \mid \mathbf{x}=\left(x, r_{h}(x, t) \cos \phi, r_{h}(x, t) \sin \phi\right), x \in[0, L], \phi \in[0,2 \pi]\right\},
\end{aligned}
$$

respectively. Observing that $d S=r_{h} \sqrt{1+r_{h, x}^{2}} d x d \phi$ is the surface element on $\Gamma_{h}$, a short calculation shows that

$$
\int_{I} r_{h}\left(\sqrt{1+r_{h, x}^{2}}-\frac{r_{h, x} r_{x}+1}{\sqrt{1+r_{x}^{2}}}\right) d x=\frac{1}{2 \pi} \int_{\Gamma_{h}}\left|\nu-\nu_{h}\right|^{2} d S .
$$

A similar relation was used in [5], [6] in an error analysis for the mean curvature flow of graphs.

(b) Under the conditions (2.13) and (3.1), the expression (3.13) is equivalent to $\left\|r_{x}-r_{h, x}\right\|_{H^{1}(I)}^{2}$. To see this, note that

$$
\begin{aligned}
& \sqrt{1+r_{h, x}^{2}}-\frac{r_{h, x} r_{x}+1}{\sqrt{1+r_{x}^{2}}} \\
& =\frac{\left(\sqrt{1+r_{h, x}^{2}} \sqrt{1+r_{x}^{2}}-\left(r_{h, x} r_{x}+1\right)\right)\left(\sqrt{1+r_{h, x}^{2}} \sqrt{1+r_{x}^{2}}+\left(r_{h, x} r_{x}+1\right)\right)}{\sqrt{1+r_{x}^{2}}\left(\sqrt{1+r_{h, x}^{2}} \sqrt{1+r_{x}^{2}}+\left(r_{h, x} r_{x}+1\right)\right)} \\
& =\frac{\left(r_{x}-r_{h, x}\right)^{2}}{\sqrt{1+r_{x}^{2}}\left(\sqrt{1+r_{h, x}^{2}} \sqrt{1+r_{x}^{2}}+\left(r_{h, x} r_{x}+1\right)\right)},
\end{aligned}
$$

which implies

$$
\begin{aligned}
\frac{c_{0}}{4\left(1+C_{0}^{2}\right) \sqrt{1+4 C_{0}^{2}}}\left\|r_{x}-r_{h, x}\right\|_{H^{1}(I)}^{2} & \leq \int_{I} r_{h}\left(\sqrt{1+r_{h, x}^{2}}-\frac{r_{h, x} r_{x}+1}{\sqrt{1+r_{x}^{2}}}\right) d x \\
& \leq C_{0}\left\|r_{x}-r_{h, x}\right\|_{H^{1}(I)}^{2}
\end{aligned}
$$

since

$$
\begin{aligned}
1 & \leq \sqrt{1+r_{x}^{2}}\left(\sqrt{1+r_{h, x}^{2}} \sqrt{1+r_{x}^{2}}+\left(r_{h, x} r_{x}+1\right)\right) \\
& \leq \sqrt{1+r_{x}^{2}}\left(\sqrt{1+r_{h, x}^{2}} \sqrt{1+r_{x}^{2}}+\sqrt{1+r_{h, x}^{2}} \sqrt{1+r_{x}^{2}}\right) \leq 2\left(1+C_{0}^{2}\right) \sqrt{1+4 C_{0}^{2}}
\end{aligned}
$$

It remains to derive an estimate for $\left\|\kappa-\kappa_{h}\right\|_{L^{2}(I)}$. 
LEMMA 3.5 .

$$
\left\|\kappa-\kappa_{h}\right\|_{L^{2}(I)} \leq C\left(\left\|r-r_{h}\right\|_{H^{1}(I)}+\left\|\kappa_{x}-\kappa_{h, x}\right\|_{L^{2}(I)}+h\right) .
$$

Proof. Clearly,

$$
\begin{aligned}
& \int_{I} r_{h}\left(\kappa-\kappa_{h}\right)^{2} d x \\
& =-\int_{I}\left(r-r_{h}\right) \kappa\left(\kappa-\kappa_{h}\right) d x+\int_{I}\left(r \kappa-r_{h} \kappa_{h}\right)\left(\kappa-I_{h} \kappa\right) d x+\int_{I}\left(r \kappa-r_{h} \kappa_{h}\right)\left(I_{h} \kappa-\kappa_{h}\right) d x .
\end{aligned}
$$

Using (3.6) in order to rewrite the third integral, we deduce

$$
\begin{aligned}
& \int_{I} r_{h}\left(\kappa-\kappa_{h}\right)^{2} d x=-\int_{I}\left(r-r_{h}\right) \kappa\left(\kappa-\kappa_{h}\right) d x+\int_{I}\left(r \kappa-r_{h} \kappa_{h}\right)\left(\kappa-I_{h} \kappa\right) d x \\
& \quad+\int_{I}\left(I_{h} \kappa-\kappa_{h}\right)\left(\sqrt{1+r_{x}^{2}}-\sqrt{1+r_{h, x}^{2}}\right) d x+\int_{I}\left(\frac{r r_{x}}{\sqrt{1+r_{x}^{2}}}-\frac{r_{h} r_{h, x}}{\sqrt{1+r_{h, x}^{2}}}\right)\left(I_{h} \kappa-\kappa_{h}\right)_{x} d x \\
& \leq C\left\|r-r_{h}\right\|_{L^{2}(I)}\left\|\kappa-\kappa_{h}\right\|_{L^{2}(I)}+C\left(\left\|r-r_{h}\right\|_{L^{2}(I)}+\left\|\kappa-\kappa_{h}\right\|_{L^{2}(I)}\right)\left\|\kappa-I_{h} \kappa\right\|_{L^{2}(I)} \\
& \quad+C\left\|I_{h} \kappa-\kappa_{h}\right\|_{L^{2}(I)}\left\|r_{x}-r_{h, x}\right\|_{L^{2}(I)}+C\left\|\left(I_{h} \kappa\right)_{x}-\kappa_{h, x}\right\|_{L^{2}(I)}\left\|r-r_{h}\right\|_{H^{1}(I)} \\
& \leq \epsilon\left\|\kappa-\kappa_{h}\right\|_{L^{2}(I)}^{2}+C_{\epsilon}\left\|r-r_{h}\right\|_{H^{1}(I)}^{2}+C_{\epsilon} h^{2}+C\left\|\kappa_{x}-\kappa_{h, x}\right\|_{L^{2}(I)}^{2} .
\end{aligned}
$$

Here we have again used (2.15). Choosing $\epsilon=\frac{c_{0}}{4}$ and recalling (3.1), we complete the proof of the lemma.

We are now in position to complete the proof of Theorem 2.3. Combining Lemmas $3.2,3.3$, and 3.5 and (3.1), we obtain with $\lambda=\frac{c_{0}}{2 \sqrt{1+4 C_{0}^{2}}}$

$$
\begin{aligned}
& \frac{1}{2} \frac{d}{d t}\left\|r-r_{h}\right\|_{L^{2}(I)}^{2}+\frac{d}{d t} \int_{I} r_{h}\left(\sqrt{1+r_{h, x}^{2}}-\frac{r_{h, x} r_{x}+1}{\sqrt{1+r_{x}^{2}}}\right) d x+\lambda\left\|\kappa_{x}-\kappa_{h, x}\right\|_{L^{2}(I)}^{2} \\
& \leq C \epsilon\left\|\kappa_{x}-\kappa_{h, x}\right\|_{L^{2}(I)}^{2}+C_{\epsilon}\left(1+\left\|r_{t}\right\|_{H^{2}(I)}\right)\left\|r-r_{h}\right\|_{H^{1}(I)}^{2}+C_{\epsilon}\left(1+\left\|r_{t}\right\|_{H^{2}(I)}^{2}\right) h^{2} .
\end{aligned}
$$

Choosing $\epsilon$ sufficiently small and recalling (3.14), the function

$$
\phi(t):=\frac{1}{2}\left\|\left(r-r_{h}\right)(t)\right\|_{L^{2}(I)}^{2}+\int_{I} r_{h}\left(\sqrt{1+r_{h, x}^{2}}-\frac{r_{h, x} r_{x}+1}{\sqrt{1+r_{x}^{2}}}\right)(t) d x
$$

satisfies

$$
\begin{gathered}
\phi^{\prime}(t)+\frac{\lambda}{2}\left\|\kappa_{x}-\kappa_{h, x}\right\|_{L^{2}(I)}^{2} \leq C\left(1+\left\|r_{t}\right\|_{H^{2}(I)}^{2}\right) h^{2}+C\left(1+\left\|r_{t}\right\|_{H^{2}(I)}\right) \phi(t), \\
0 \leq t \leq \hat{T}_{h}
\end{gathered}
$$

Now, (2.7) and (3.14) yield $\phi(0) \leq C h^{2}$, so that Gronwall's lemma implies

$$
\phi(t) \leq C h^{2}\left(1+\int_{0}^{T}\left\|r_{t}\right\|_{H^{2}(I)}^{2} d t\right) \exp \left(\int_{0}^{T} C\left(1+\left\|r_{t}\right\|_{H^{2}(I)}\right) d t\right), \quad 0 \leq t \leq \hat{T}_{h} .
$$

Therefore,

$$
\sup _{0<t<\hat{T}_{h}}\left\|\left(r-r_{h}\right)(t)\right\|_{H^{1}(I)} \leq C h
$$


and, using (3.15) together with Lemma 3.5,

$$
\int_{0}^{\hat{T}_{h}}\left\|\kappa-\kappa_{h}\right\|_{H^{1}(I)}^{2} d t \leq C h^{2} .
$$

We can now prove that $\hat{T}_{h}=T$. If not, we would have $\hat{T}_{h}<T$; the smoothness of $r$, (3.16), and an inverse estimate then would imply that

$$
\left\|\left(r-r_{h}\right)(t)\right\|_{H^{1, \infty}(I)} \leq C \sqrt{h}, \quad 0 \leq t \leq \hat{T}_{h},
$$

which combined with (2.13) would give

$$
\frac{3}{4} c_{0} \leq r_{h} \leq \frac{3}{2} C_{0},\left|r_{h, x}\right| \leq \frac{3}{2} C_{0} \quad \text { in } I \times\left[0, \hat{T}_{h}\right]
$$

provided that $h \leq h_{0}$ and $h_{0}$ is sufficiently small. However, then we could extend the discrete solution to an interval $\left[0, \hat{T}_{h}+\delta\right]$ for some $\delta>0$ with

$$
\frac{1}{2} c_{0} \leq r_{h} \leq 2 C_{0},\left|r_{h, x}\right| \leq 2 C_{0} \quad \text { in } I \times\left[0, \hat{T}_{h}+\delta\right],
$$

which contradicts the definition of $\hat{T}_{h}$. Thus $\hat{T}_{h}=T$ for $h \leq h_{0}$ and (3.16), (3.17) imply our result.

4. Numerical results. We use the notation

$$
\begin{aligned}
& r_{j}(t)=r_{h}\left(x_{j}, t\right), \quad \kappa_{j}(t)=\kappa_{h}\left(x_{j}, t\right), \quad j=0, \ldots, N, \\
& q_{j}(t)=\sqrt{h_{j}^{2}+\left(r_{j}(t)-r_{j-1}(t)\right)^{2}}, \quad j=1, \ldots, N .
\end{aligned}
$$

The spatially discrete problem (2.5), (2.6) then is translated into the following system of ODEs. By a dot we denote the time derivative. For numerical tests we shall use an additional right-hand side $f$ which we include in the equations here.

$$
\begin{aligned}
\frac{h_{j}}{6}\left(r_{j-1}+r_{j}\right) \dot{r}_{j-1} & +\left(\frac{h_{j}}{6} r_{j-1}+\frac{1}{2}\left(h_{j}+h_{j+1}\right) r_{j}+\frac{h_{j+1}}{6} r_{j+1}\right) \dot{r}_{j} \\
& +\frac{h_{j+1}}{6}\left(r_{j}+r_{j+1}\right) \dot{r}_{j+1}-\frac{r_{j-1}+r_{j}}{q_{j}} \kappa_{j-1}+\left(\frac{r_{j-1}+r_{j}}{q_{j}}+\frac{r_{j}+r_{j+1}}{q_{j+1}}\right) \kappa_{j} \\
& -\frac{r_{j}+r_{j+1}}{q_{j+1}} \kappa_{j+1}=\frac{1}{2}\left(q_{j}\left(r_{j-1}+r_{j}\right) f_{j-\frac{1}{2}}+q_{j+1}\left(r_{j}+r_{j+1}\right) f_{j+\frac{1}{2}}\right), \\
\frac{h_{j}}{6}\left(r_{j-1}+r_{j}\right) \kappa_{j-1} & +\left(\frac{h_{j}}{6} r_{j-1}+\frac{1}{2}\left(h_{j}+h_{j+1}\right) r_{j}+\frac{h_{j+1}}{6} r_{j+1}\right) \kappa_{j} \\
& +\frac{h_{j+1}}{6}\left(r_{j}+r_{j+1}\right) \kappa_{j+1}+\frac{r_{j-1}+r_{j}}{q_{j}} r_{j-1}-\left(\frac{r_{j-1}+r_{j}}{q_{j}}+\frac{r_{j}+r_{j+1}}{q_{j+1}}\right) r_{j} \\
& +\frac{r_{j}+r_{j+1}}{q_{j+1}} r_{j+1}=q_{j}+q_{j+1}
\end{aligned}
$$

for $j=1, \ldots, N, t \in(0, T]$, with periodic boundary conditions and initial condition $r_{j}(0)=r_{0}\left(x_{j}\right), j=0, \ldots, N$. For the right-hand side term involving $f$ we have used a simple integration formula and the notation $f_{j \pm \frac{1}{2}}=f\left(\left(x_{j}+x_{j \pm 1}\right) / 2\right)$. 
The time discretization is done via a semi-implicit scheme which also linearizes the problem. Furthermore we use mass lumping at suitable positions. Let $\tau>0$ be the time step size and $M=[T / \tau]$. For a generic function $w$ we denote by $w^{m}$ $(0 \leq m \leq M)$ the evaluation on the $m$ th time level: $w^{m}=w(\cdot, m \tau)$. The fully discrete scheme then reads as follows.

Algorithm 4.1. Let $r_{j}^{0}=r_{0}\left(x_{j}\right), j=0, \ldots, N$. For $m=1, \ldots, M$ solve

$$
\begin{aligned}
\frac{1}{\tau}\left(h_{j}+h_{j+1}\right) r_{j}^{m-1}\left(r_{j}^{m}-r_{j}^{m-1}\right) & \\
-\frac{r_{j-1}^{m-1}+r_{j}^{m-1}}{q_{j}^{m-1}} \kappa_{j-1}^{m}+ & \left(\frac{r_{j-1}^{m-1}+r_{j}^{m-1}}{q_{j}^{m-1}}+\frac{r_{j}^{m-1}+r_{j+1}^{m-1}}{q_{j+1}^{m-1}}\right) \kappa_{j}^{m}-\frac{r_{j}^{m-1}+r_{j+1}^{m-1}}{q_{j+1}^{m-1}} \kappa_{j+1}^{m} \\
& =\frac{1}{2}\left(q_{j}^{m-1}\left(r_{j-1}^{m-1}+r_{j}^{m-1}\right) f_{j-\frac{1}{2}}^{m}+q_{j+1}^{m-1}\left(r_{j}^{m-1}+r_{j+1}^{m-1}\right) f_{j+\frac{1}{2}}^{m}\right), \\
\left(h_{j}+h_{j+1}\right) r_{j}^{m-1} \kappa_{j}^{m} & \\
+\frac{r_{j-1}^{m-1}+r_{j}^{m-1}}{q_{j}^{m-1}} r_{j-1}^{m}- & \left(\frac{r_{j-1}^{m-1}+r_{j}^{m-1}}{q_{j}^{m-1}}+\frac{r_{j}^{m-1}+r_{j+1}^{m-1}}{q_{j+1}^{m-1}}\right) r_{j}^{m}+\frac{r_{j}^{m-1}+r_{j+1}^{m-1}}{q_{j+1}^{m-1}} r_{j+1}^{m} \\
& =q_{j}^{m-1}+q_{j+1}^{m-1}
\end{aligned}
$$

for $j=1, \ldots, N, m=1, \ldots, M$.

In every time step a linear system for $\underline{r}^{m}=\left(r_{1}^{m}, \ldots, r_{N}^{m}\right)$ and $\underline{\kappa}^{m}=\left(\kappa_{1}^{m}, \ldots, \kappa_{N}^{m}\right)$ of the form

$$
\begin{gathered}
\frac{1}{\tau} M^{m-1} \underline{r}^{m}+S^{m-1} \underline{\kappa}^{m}=\underline{c}^{m-1}, \\
M^{m-1} \underline{\kappa}^{m}-S^{m-1} \underline{r}^{m}=\underline{d}^{m-1}
\end{gathered}
$$

has to be solved. Here $M^{m-1}$ is a suitable mass matrix, $S^{m-1}$ is a stiffness matrix, and $\underline{c}^{m-1}, \underline{d}^{m-1}$ are right-hand sides depending on the quantities of the $(m-1)$ st time step with built-in periodic boundary conditions. Note that the time discretization is semiimplicit with respect to the position $r$ but is fully implicit with respect to curvature $\kappa$. The linear system (4.2), (4.3) was solved by inserting the second equation into the first one, which leads to the following linear system for $\underline{r}^{m}$ :

$$
\left(\frac{1}{\tau} M^{m-1}+S^{m-1}\left(M^{m-1}\right)^{-1} S^{m-1}\right) \underline{r}^{m}=\underline{c}^{m-1}-S^{m-1}\left(M^{m-1}\right)^{-1} \underline{d}^{m-1}
$$

Note that the matrix $M^{m-1}$ is a diagonal matrix. The system (4.4) was solved by a conjugate gradient method.

For all computations we have used uniform spatial grids $h_{j}=h$ with $h$ as indicated.

We test the scheme with a known continuous solution. We choose

$$
r(x, t)=(1+0.25 \sin \pi(x-1))(1+0.125 \cos t)
$$

on the interval $I=[0,2]$ for $T=1$ and calculate the corresponding right-hand side $f$ from (1.3) and (1.2). Now we are able to compute the error between continuous solution $r, \kappa$ and discrete solution $r_{h}^{m}, \kappa_{h}^{m}$ and calculate the experimental order of convergence from the errors for two grids. As time step size we have chosen $\tau=0.1 h^{2}$. 
TABLE 4.1

Absolute errors in various norms and experimental orders of convergence (in brackets) for the test problem for the choice $\tau=0.1 h^{2}$.

\begin{tabular}{|l|l|l|l|}
\hline$N$ & $h$ & $\left\|r-r_{h}\right\|_{L^{\infty}\left(H^{1}\right)}$ & $\left\|\kappa-\kappa_{h}\right\|_{L^{2}\left(H^{1}\right)}$ \\
\hline 20 & 0.1 & 0.3010 & 2.2669 \\
40 & 0.05 & $0.1544(0.96)$ & $1.1693(0.96)$ \\
80 & 0.025 & $0.07784(0.99)$ & $0.5892(0.99)$ \\
160 & 0.0125 & $0.03903(1.00)$ & $0.2952(1.00)$ \\
320 & 0.00625 & $0.01953(1.00)$ & $0.1477(1.00)$ \\
\hline
\end{tabular}

TABLE 4.2

Absolute errors in various norms and experimental orders of convergence (in brackets) for the test problem for the choice $\tau=0.1 \mathrm{~h}$.

\begin{tabular}{|l|l|l|l|}
\hline$N$ & $h$ & $\left\|r-r_{h}\right\|_{L^{\infty}\left(H^{1}\right)}$ & $\left\|\kappa-\kappa_{h}\right\|_{L^{2}\left(H^{1}\right)}$ \\
\hline 20 & 0.1 & 0.2575 & 2.2597 \\
40 & 0.05 & $0.1399(0.88)$ & $1.1672(0.95)$ \\
80 & 0.025 & $0.07363(0.93)$ & $0.5886(0.99)$ \\
160 & 0.0125 & $0.03790(0.96)$ & $0.2950(1.00)$ \\
320 & 0.00625 & $0.01922(0.98)$ & $0.1476(1.00)$ \\
\hline
\end{tabular}

The results are shown in Table 4.1. We measured the errors

$$
\left\|r-r_{h}\right\|_{L^{\infty}\left((0, T), H^{1}(I)\right)} \quad \text { and } \quad\left\|\kappa-\kappa_{h}\right\|_{L^{2}\left((0, T), H^{1}(I)\right)} .
$$

The results confirm the error estimates in Theorem 2.3 precisely. A quite astonishing result is that these convergence results experimentally also hold in the case of linear coupling of time step size and spatial grid size (see Table 4.2), in particular, that no stability problems arise even though the scheme is only semi-implicit. This is in some sense similar to the case of mean curvature flow, for which in [7] stability of a semi-implicit scheme was proved without any time step restriction.

In [3] it was shown that solutions of axially symmetric surface diffusion may exhibit the following dynamical behavior: After an initial rapid decay, some perturbations slowly grow in amplitude and finally lead to pinch-off. We recomputed an example from [3], for which the initial surface is given by

$$
r_{0}(x)=1+0.05\left(\sin \left(\frac{m+1}{2} x\right)+\sin \left(\frac{m}{2} x\right)\right), x \in(0, n \pi) .
$$

Figure 4.1 shows the rapid decay of perturbations for $m=10$. For better visibility we scaled the graphics vertically by 100 .

For $m=14$ we show the long time behavior of the solution $r=r(x, t)$. In order to make the dynamical behavior more transparent we plot the solution in Figure 4.2 for $t \in[0,10]$ and in Figure 4.3 for $t \in[20,27.861]$. We have used 400 nodes and a time step size $\tau=0.1 h^{2}$. Note that our error analysis is only valid as long as $r$ is bounded away from zero. For calculations near the pinch-off singularity we adapted the time step according to $\tau=0.1 h^{2} \min _{[0,4 \pi]} r_{h}^{3}$, a criterion which was found experimentally. Finally, we computed the solution of axisymmetric surface diffusion for the initial surface given by

$$
r_{0}(x)=1-0.95|x| \sin \frac{\pi}{x}, x \in(-1,1)
$$

Here we have used 500 spatial nodes and a time discretization as in the previous example. The results are shown in Figure 4.4. 

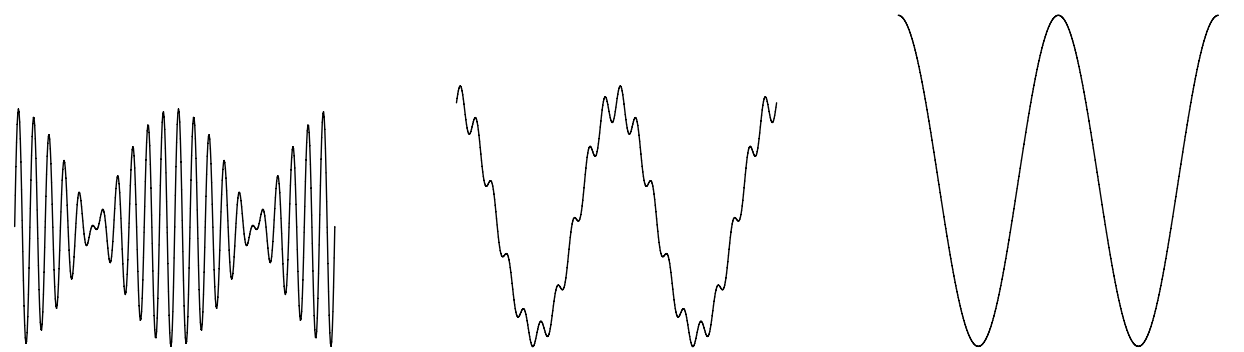

FIG. 4.1. Evolution of the initial surface given by (4.5) with $m=10, n=8$ for $t=0.0,0.01$, 0.1 , vertically scaled by 100 .
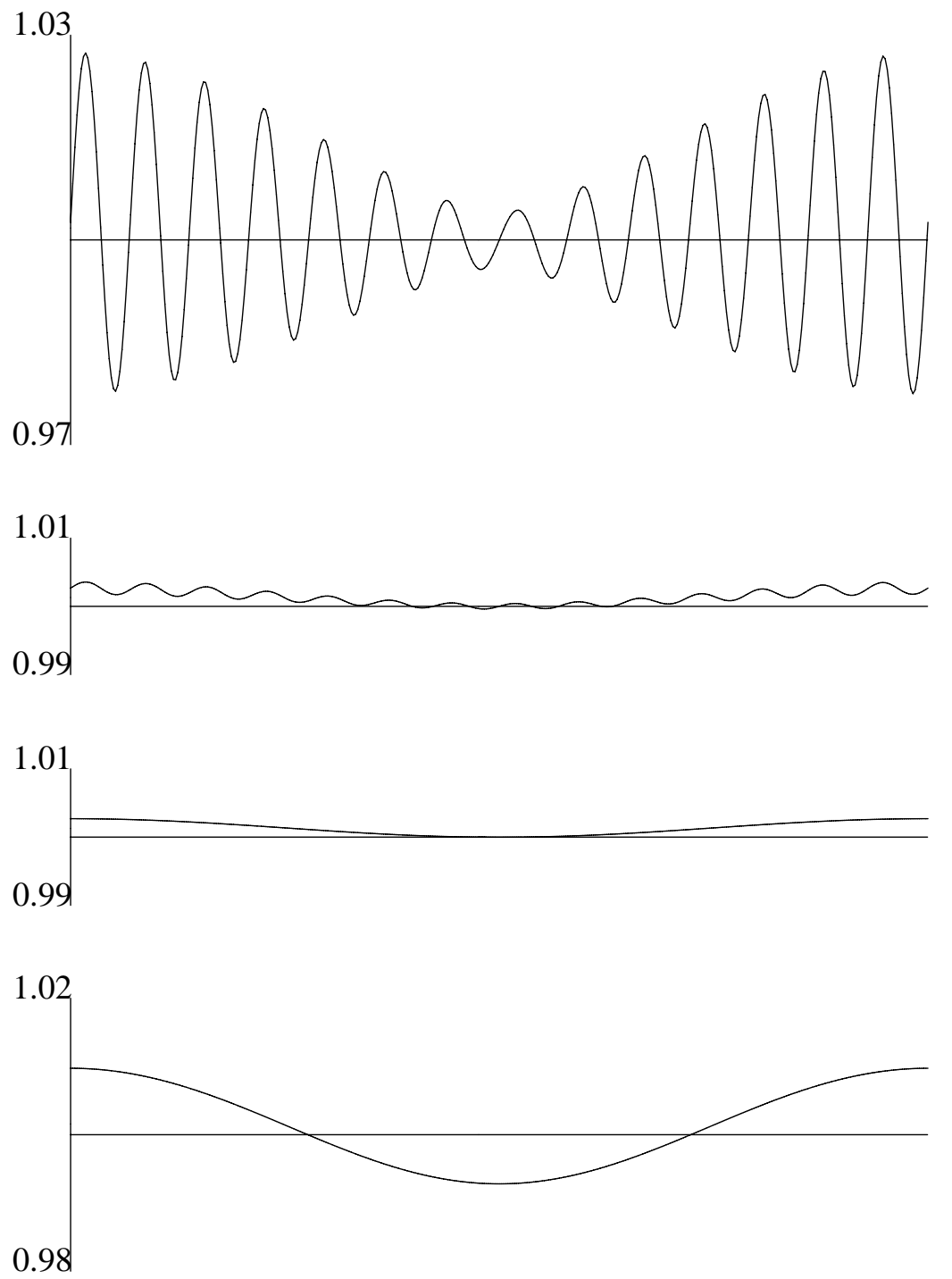

FIG. 4.2. Evolution of the axially symmetric initial surface given by (4.5) with $m=14, n=4$ under surface diffusion. The horizontal axis runs from 0 to $4 \pi$, and the vertical axis is scaled by 100. Time steps $t=0.00,0.0014,0.10$, and 10.0 . 

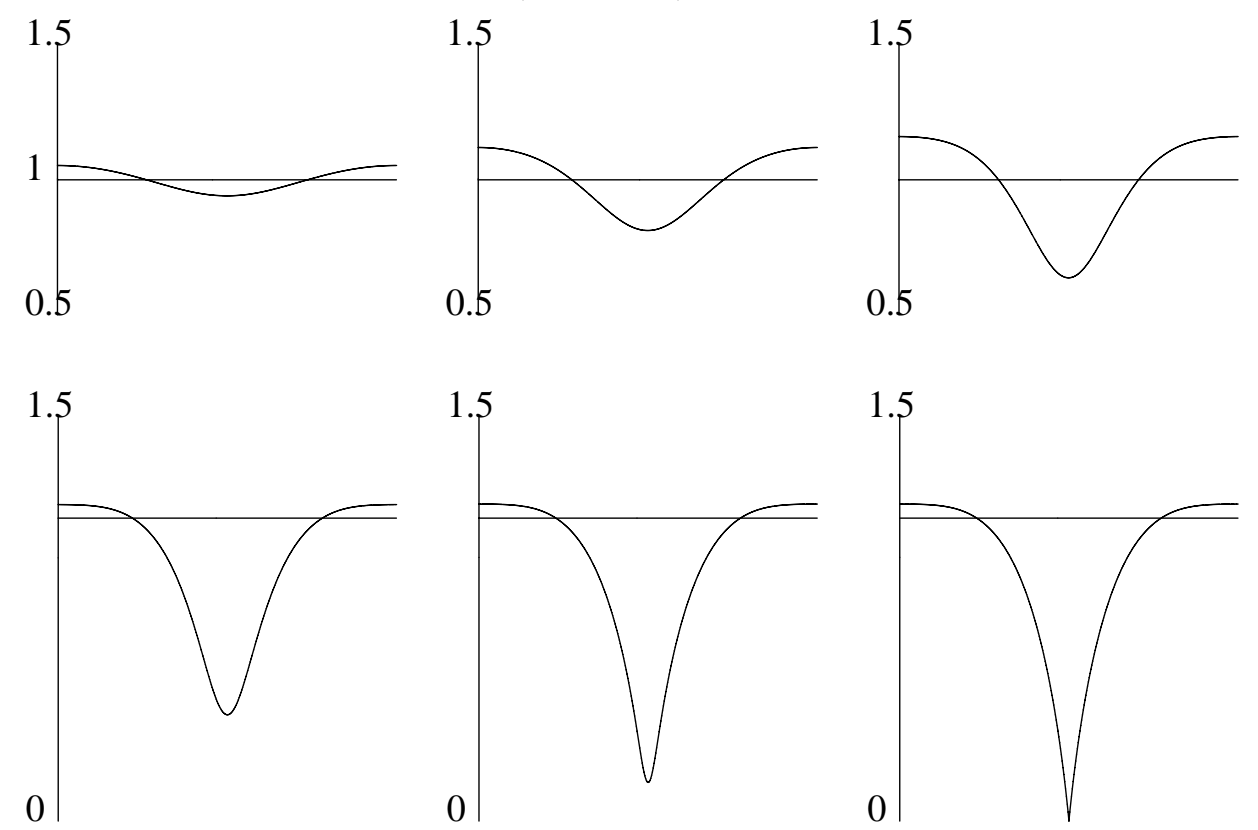

FIG. 4.3. Evolution of the axially symmetric initial surface given by (4.5) with $m=14, n=4$ under surface diffusion. The horizontal axis runs from 0 to $4 \pi$, and the vertical axis is scaled by 10 . Time steps $t=20.0,25.0,27.0,27.75,27.86$, and 27.861 .
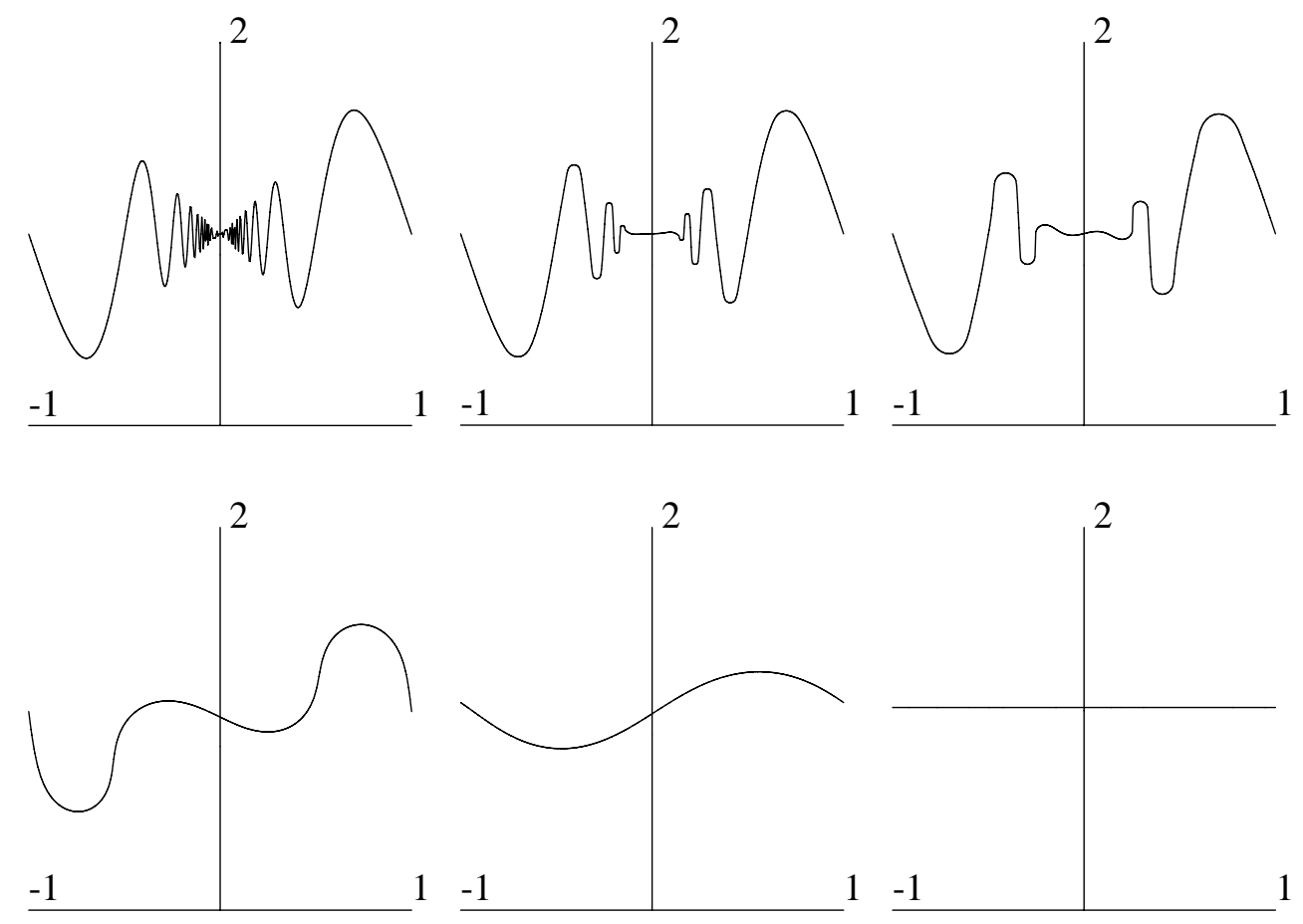

FIG. 4.4. Evolution of the axially symmetric initial surface given by (4.6) under surface diffusion. Time steps $t=0.00,6.26 \cdot 10^{-7}, 7.59 \cdot 10^{-6}, 6.97 \cdot 10^{-4}, 6.45 \cdot 10^{-3}$, and $9.82 \cdot 10^{-2}$. 


\section{REFERENCES}

[1] A. J. Bernoff, A. L. Bertozzi, and T. P. Witelski, Axisymmetric surface diffusion: Dynamics and stability of self-similar pinchoff, J. Statist. Phys., 93 (1998), pp. 725-776.

[2] J. W. Cahn and J. E. Taylor, Surface motion by surface diffusion, Acta Metall. Mater., 42 (1994), pp. 1045-1063.

[3] B. D. Coleman, R. S. Falk, And M. Moakher, Stability of cylindrical bodies in the theory of surface diffusion, Phys. D, 89 (1995), pp. 123-135.

[4] B. D. Coleman, R. S. Falk, and M. Moakher, Space-time finite element methods for surface diffusion with applications to the theory of the stability of cylinders, SIAM J. Sci. Comput., 17 (1996), pp. 1434-1448.

[5] K. Deckelnick And G. Dziuk, Discrete anisotropic curvature flow of graphs, M2AN Math. Model. Numer. Anal., 33 (1999), pp. 1203-1222.

[6] K. Deckelnick and G. Dziuk, Error estimates for a semi implicit fully discrete finite element scheme for the mean curvature flow of graphs, Interfaces Free Bound., 2 (2000), pp. 341359.

[7] G. DzIUK, Numerical schemes for the mean curvature flow of graphs, in Proceedings of the IUTAM Symposium on Variations of Domains and Free-Boundary Problems in Solid Mechanics, P. Argoul, M. Frémond, and Q. S. Nguyen, eds., Kluwer Academic Publishers, Dordrecht, Boston, London, 1999, pp. 63-70.

[8] C. M. Elliott, D. A. French, and F. A. Milner, A second order splitting method for the Cahn-Hilliard equation, Numer. Math., 54 (1989), pp. 575-590.

[9] C. M. Elliott and H. Garcke, Existence results for diffusive surface motion laws, Adv. Math. Sci. Appl., 7 (1997), pp. 467-490.

[10] J. Escher, U. F. MAYER, AND G. Simonett, The surface diffusion flow for immersed hypersurfaces, SIAM J. Math. Anal., 29 (1998), pp. 1419-1433.

[11] Y. Giga AND K. ITo, On pinching of curves moved by surface diffusion, Commun. Appl. Anal., 2 (1998), pp. 393-405.

[12] C. Herring, Surface diffusion as a motivation for sintering, in The Physics of Powder Metallurgy, W. E. Kingston, ed., McGraw-Hill, New York, 1951, pp. 143-179.

[13] U. F. Mayer And G. Simonett, Self-intersections for the surface diffusion and the volumepreserving mean curvature flow, Differential Integral Equations, 13 (2000), pp. 1189-1199.

[14] W. W. Mullins, Theory of thermal grooving, J. Appl. Phys., 28 (1957), pp. 333-339.

[15] F. A. Nichols And W. W. Mullins, Surface-(interface-) and volume-diffusion contributions to morphological changes driven by capillarity, Trans. Metall. Soc., AIME, 233 (1965), pp. $1840-1847$.

[16] H. Wong, M. J. Miksis, P. W. Voorhees, And S. H. Davis, Universal pinch off of rods by capillarity-driven surface diffusion, Scripta Mat., 39 (1998), pp. 55-60. 